\title{
Diseño de un panel multicolor para evaluar moléculas intracelulares y de superficie mediante citometría de flujo
}

\author{
Jose Mateus ${ }^{1,2^{*}}$, Paola Lasso ${ }^{1,2^{*}}$, John Mario González ${ }^{3}$, Concepción Judith Puerta², Adriana Cuéllar ${ }^{1}$ \\ Grupo de Inmunobiología y Biología Celular, Pontificia Universidad Javeriana, Bogotá, D.C., Colombia \\ 2 Laboratorio de Parasitología Molecular, Pontificia Universidad Javeriana, Bogotá, D.C., Colombia \\ ${ }^{3}$ Grupo de Ciencias Básicas Médicas, Facultad de Medicina, Universidad de los Andes, Bogotá, D.C., Colombia \\ * Los autores contribuyeron de igual manera en el presente estudio. \\ Institución donde se llevó a cabo el trabajo: Pontificia Universidad Javeriana, Bogotá, Colombia.
}

Introducción. La citometría de flujo permite detectar la presencia de moléculas intracelulares y de superficie, de forma simultánea sobre cada célula.

Objetivo. Describir un método para la construcción armónica de un panel multicolor con 11 parámetros para el análisis fenotípico y funcional de linfocitos T (LT) CD8+ por citometría de flujo.

Materiales y métodos. Para la construcción del panel multicolor, se seleccionaron las moléculas y se titularon los conjugados con fluorocromos para la determinación de CD3, CD8, CCR7, CD28, CD27, CD45RA, CD95 y CD127, en células mononucleares de sangre periférica. Para la evaluación del panel, se hizo la construcción progresiva adicionando uno a uno los conjugados y la fluorescencia menos uno (FMO). Este método fue aplicado para células ex vivo y para evaluar la producción de IFN $\gamma$, IL-2 y TNFa frente al estímulo con la enterotoxina B de Staphylococcus aureus (SEB) y al antígeno crudo de Trypanosoma cruzi. Finalmente, se procedió al análisis de las subpoblaciones de LT CD8 ${ }^{+}$ex vivo en individuos sanos.

Resultados. La evaluación de las moléculas con los conjugados no mostró interferencia en las señales de fluorescencia. Las frecuencias de las subpoblaciones de LT CD8 ${ }^{+}$evaluadas fueron cercanas a los valores reportados en otros estudios. Además, se observó que la frecuencia de LT CD8 ${ }^{+}$productores de IFN $\gamma$, IL-2 y TNFa fue mayor a las seis horas de cultivo con SEB y con el antígeno crudo de $T$. cruzi.

Conclusiones. El método aplicado para la construcción del panel multicolor permite obtener frecuencias de las subpoblaciones de LT CD8 ${ }^{+}$que corresponden a lo reportado en la literatura científica.

Palabras clave: citometría de flujo, colorantes fluorescentes, subpoblaciones linfocitarias, linfocitos T, citocinas, inmunofenotipificación.

doi: http://dx.doi.org/10.7705/biomedica.v33i4.1709

Design of a multicolor panel to assess intracellular and surface molecules by flow cytometry

Introduction: Flow cytometry allows simultaneous detection of surface and intracellular molecules on each cell.

Objective: To describe a method for building up a harmonic multicolor panel with 11 flow cytometry parameters for phenotypic and functional analysis on CD8 ${ }^{+} \mathrm{T}$ lymphocytes.

Materials and methods: For the multicolor panel construction, we selected the molecules and titred conjugated antibodies with fluorochromes for CD3, CD8, CCR7, CD28, CD27, CD45RA, CD95 and CD127 determination in peripheral blood mononuclear cells (PBMC). To evaluate the panel, the conjugated antibodies were gradually added one by one and fluorescence minus one (FMO) test was performed. This method was applied to assess ex vivo subpopulations of T cells and the production of intracellular IFN $\gamma$, IL-2 and TNFa using polyclonal stimulation with enterotoxin B from Staphylococcus aureus (SEB) and antigen-specific cells with crude Trypanosoma cruzi antigen. Finally, the ex vivo $\mathrm{CD}^{+} \mathrm{T}$ lymphocyte subpopulations frequencies were analyzed in healthy individuals.

Results: The evaluation of the selected molecules and conjugates did not show interference in the fluorescence signals and detection. The frequencies of $\mathrm{CD}^{+} \mathrm{T}$ cells evaluated were similar to the values reported in other studies. Additionally, we observed that the frequency of CD8 ${ }^{+} \mathrm{T}$ lymphocytes

\footnotetext{
Contribución de los autores:

Jose Mateus y Paola Lasso realizaron los experimentos y elaboraron el manuscrito.

John Mario González y Concepción Judith Puerta participaron en el diseño del estudio.

Adriana Cuéllar: diseño y supervisión del estudio, y obtención el apoyo financiero.

Todos los autores participaron en el análisis de los datos, leyeron y aprobaron el manuscrito.
} 
producing IFN $\gamma$, IL-2 and TNFa was higher 6 hours after culture with SEB and crude T. cruzi lysate. Conclusions: The method used for the construction of a multicolor panel allows obtaining frequencies of $\mathrm{CD}^{+} \mathrm{T}$ lymphocyte subpopulations corresponding to those reported in the literature.

Key words: Flow cytometry, fluorescent dyes, lymphocyte subsets, T-lymphocytes, cytokines, immunophenotyping.

doi: http://dx.doi.org/10.7705/biomedica.v33i4.1709

La citometría de flujo como técnica analítica ha sido utilizada desde finales de 1960 en el laboratorio de los esposos Herzenberg en Stanford University en Estados Unidos y actualmente es una de las tecnologías de evaluación celular más poderosa empleada para el análisis celular $(1,2)$.

El análisis multiparamétrico permite medir sobre una misma célula diversos parámetros, como tamaño, complejidad y presencia de moléculas intracelulares o extracelulares, mediante su marcación con anticuerpos conjugados con fluorocromos. Los primeros citómetros de flujo permitían analizar tres parámetros, que incluían una señal fluorescente y dos señales de dispersión de luz. La necesidad de analizar fenotípica y funcionalmente subpoblaciones celulares, condujo a mejorar la tecnología y fue así como a mediados de 1980 se hicieron los primeros análisis de seis parámetros, cuatro señales fluorescentes y dos señales de dispersión de luz. En 1990, los avances en citometría de flujo permitieron ahondar en la complejidad del sistema inmunitario y fue así como se logró el análisis de 10 a 17 parámetros por célula, lo que permitió identificar cientos de fenotipos celulares diferentes así como los asociados a diversas funciones de cada subpoblación (3-5). Por lo tanto, el incremento en el número de canales de detección y la variabilidad de marcadores y anticuerpos conjugados a fluorocromos disponibles, han permitido crear paneles multicolor que evalúan gran cantidad de parámetros de manera simultanea (5). Además, el análisis de alta velocidad es otra de las ventajas de esta tecnología, con la posibilidad de evaluar más de 10.000 eventos por segundo $(6,7)$.

El aumento en la complejidad de los análisis, requiere la introducción de métodos de control que

\footnotetext{
Correspondencia:

Adriana Cuéllar, Facultad de Ciencias, Pontificia Universidad Javeriana, Carrera $7 \mathrm{~N}^{\circ}$ 43-82, Edificio Carlos Ortiz 52, oficina 608, Bogotá, D.C., Colombia.

Teléfono: (571) 320 8320, extensión 4072; fax: (571) 3208320 , extensión 4021

acuellar@javeriana.edu.co

Recibido: 17/12/12; aceptado:26/06/13
}

permitan lograr la construcción de paneles multicolor para un análisis adecuado de las poblaciones celulares de interés, evitando interferencias 0 pérdida de sensibilidad por autofluorescencia o comportamientos no esperados en la combinación de fluorocromos $(6,8)$.

Nuestro grupo de investigación ha venido trabajando en la respuesta inmunitaria de la enfermedad de Chagas, causada por el parásito intracelular Trypanosoma cruzi. Se han logrado avances importantes en el estudio de parámetros inmunológicos mediante el uso de la citometría de flujo (9-12). En modelos de ratón de la infección por $T$. cruzi se ha mostrado que la eliminación de la población de los linfocitos T (LT) $\mathrm{CD}^{+}$conlleva la muerte de los animales (13). En humanos infectados crónicamente, se ha demostrado la presencia de $\mathrm{LT} \mathrm{CD8}^{+}$específicos del parásito, células importantes en la eliminación de antígenos intracelulares (14).

Esta nota técnica muestra un método para la construcción y evaluación de paneles multicolor para el análisis fenotípico y funcional de LT CD8 ${ }^{+}$ en células ex vivo, y en respuesta a un estímulo policlonal o específico con base en metodologías previamente descritas $(6,8,15,16)$.

\section{Materiales y métodos}

El diseño experimental propuesto para la marcación de moléculas en células ex vivo y cultivadas, involucra las siguientes etapas: selección de moléculas y conjugados, titulación de conjugados y evaluación del panel multicolor a partir de células obtenidas de individuos sanos. Para incluir parámetros de validación en el modelo de interés, se evaluó la producción de citocinas en células de pacientes con enfermedad de Chagas.

\section{Obtención de células}

Las células mononucleares de sangre periférica se obtuvieron por gradiente de densidad con FicollHypaque (GE Healthcare; Uppsala, Suecia), a partir de sangre periférica anticoagulada con heparina de sodio (BD Vacutainer; Franklin Lakes, NJ, USA). Para la titulación de los conjugados y 
la evaluación de los paneles, se incluyeron dichas células de un individuo sano. Para el estudio de las subpoblaciones de $\mathrm{LT} \mathrm{CD}^{+}$, se incluyeron cinco individuos sanos. Para el análisis de la producción de citocinas (IFN $\gamma$, IL-2 y TNFa), se incluyeron células mononucleares de sangre periférica de cinco individuos con serología positiva para anticuerpos específicos de $T$. cruzi por ELISA e inmunofluorescencia indirecta (IFI), con diagnóstico clínico de enfermedad de Chagas en fase crónica sintomática. Los individuos incluidos aceptaron participar voluntariamente y firmaron el consentimiento informado. Este estudio fue avalado por los Comités de Ética de la Pontificia Universidad Javeriana y el Hospital Universitario San Ignacio.

\section{Marcación de moléculas en células ex vivo}

\section{Selección de moléculas y conjugados}

La selección de los fluorocromos para los anticuerpos conjugados se basa en la expresión del antígeno sobre las células de interés y el índice de coloración del fluorocromo (17). Este último permite establecer la capacidad de un fluorocromo para discriminar entre una señal positiva débil y el ruido de fondo, es decir, establece la sensibilidad de resolución.

Los conjugados para cada molécula, se seleccionaron teniendo en cuenta los siguientes aspectos:

1) se seleccionaron los fluorocromos menos brillantes para aquellos antígenos que permiten identificar subpoblaciones celulares y separan claramente las poblaciones positivas de las negativas (antígenos primarios: CD3, CD8);

2) losfluorocromosconbrillo intermedioseutilizaron para antígenos que caracterizan poblaciones celulares, pero su patrón de expresión es un continuo (antígenos secundarios: CD45RA, CD28); $y$

3) los fluorocromos con mayor sensibilidad de resolución, se usaron para aquellos antígenos con bajo nivel de expresión, eventos poco frecuentes o que no se encuentran bien caracterizados (antígenos terciarios: IFN $\gamma$, IL-2) (6).

Los conjugados seleccionados para el análisis ex vivo (panel 1) fueron: CD3-Pacific Blue (BD Pharmingen; clon UCHT1; Catálogo No. 558117; San Diego, CA, USA), CD8-APC H7 (BD Biosciences; clon SK1; Catálogo No. 641400; San
Jose, CA, USA), CD45RA-PE (BD Pharmingen; clon HI100; Catálogo No. 555489), CCR7-PE-Cy7 (BD Pharmingen; clon 3D12; Catálogo No. 557648), CD28-PerCP-Cy5.5 (BD Biosciences; Clon L293; Catálogo No. 337181), CD27-Alexa Fluor 700 (BD Pharmingen; Clon M-T271; Catálogo No. 560611), CD95-APC (BD Pharmingen; Clon DX2; Catálogo No. 558814) y CD127-FITC (BD Pharmingen; Clon HIL-7R-M21; Catálogo No. 560549). Además, se incluyó el marcador de viabilidad Fixable Aqua Dead Cell Stain (Invitrogen; Catálogo No. L34957; Eugene, OR, USA).

\section{Titulación de conjugados}

Para la titulación de los conjugados, se hicieron cinco diluciones seriadas 1:2 de cada conjugado, partiendo de la concentración recomendada por la casa comercial (8). Posteriormente, un millón de células mononucleares de sangre periférica se incubaron en la oscuridad durante 30 minutos a 4 C, con las concentraciones de cada anticuerpo conjugado. Para disminuir la unión inespecífica entre las células y los conjugados, las células se lavaron con la solución tampón fosfato-alcalino para tinción (PBS 1X, PBS 0,01 M, pH 7,4) (Eurobio; Les Ulis, Francia) con suero fetal bovino al $1 \%$. Para cada concentración se obtuvo el índice de tinción, dado por el cociente entre la intensidad media de fluorescencia de la población positiva para la expresión del marcador y la intensidad media de fluorescencia de la población negativa. En cada uno de los casos se seleccionó el punto de saturación con base en el mejor índice de tinción, que representa la mayor separación entre la población positiva y la negativa. Además, se seleccionó la concentración de conjugado que permite la mejor distribución de las poblaciones, con respecto a los ejes en las gráficas de puntos o dispersogramas. Finalmente, la frecuencia de la población celular obtenida con la concentración seleccionada del conjugado, se comparó con las frecuencias reportadas en otros estudios $(5,8)$.

\section{Evaluación del panel multicolor}

Una vez seleccionados los marcadores y fluorocromos por utilizar, se procedió a hacer el montaje progresivo del panel con el fin de evaluar posibles interferencias entre los fluorocromos usados. Paraello, unmillóndecélulasmononucleares de sangre periférica se incubaron en la oscuridad durante 30 minutos a $4{ }^{\circ} \mathrm{C}$ con los conjugados previamente titulados y, posteriormente, se lavaron con la solución tampón para tinción. En el primer tubo se incubaron las células con el marcador 
de viabilidad, y en el siguiente tubo se adicionó, además del marcador de viabilidad, el conjugado para CD3. De esta manera, se adicionaron uno a uno todos los anticuerpos conjugados a cada tubo, en el siguiente orden: anti-CD8, seguido por anti-CCR7, anti-CD28, anti-CD27, anti-CD45RA, anti-CD95 y anti-CD127. La interferencia de los conjugados se evaluó con base en que la adición progresiva de un anticuerpo conjugado pudiese modificar la señal de los conjugados agregados anteriormente.

El panel también fue evaluado por el control de fluorescencia menos uno (Fluorescence Minus One, $F M O$ ), en la cual para evaluar las interferencias producidas por determinados conjugados, se marcaron las células con todos los conjugados menos el conjugado por evaluar, incubando un millón de células mononucleares en la oscuridad durante 30 minutos a $4{ }^{\circ} \mathrm{C}$, y luego lavándolas con la solución tampón para tinción. Por ejemplo, la fluorescencia menos uno para la molécula CD45RA se hizo marcando las células con todos los anticuerpos conjugados antes mencionados, menos el conjugado para CD45RA. De la misma forma se procedió para cada una de las moléculas. En el control fluorescencia menos uno, la interferencia de un anticuerpo conjugado específico se observa por la presencia de fluorescencia en el canal de detección del conjugado no adicionado.

\section{Marcación para la evaluación de citocinas intracelulares en células cultivadas}

Obtención del antígeno crudo de tripomastigotes de Trypanosoma cruzi

La obtención de tripomastigotes de $T$. cruzi se hizo en células VERO (ATCC CCL-81, Manassas, VA, USA), según lo reportado por Yoshida, et al. (18), con algunas modificaciones. Brevemente, formas metacíclicas de la cepa de T. cruzi I MHOM/CO/01/ DA se incubaron 24 horas con células VERO a $37^{\circ} \mathrm{C}$ y $5 \%$ de $\mathrm{CO}_{2}$ en medio Dulbecco's Modified Eagle Medium (DMEM, Eurobio) con suplemento de suero fetal bovino al $10 \%$ en una relación parásito/célula de 10:1. El antígeno crudo de tripomastigotes de $T$. cruzi se obtuvo de acuerdo con lo descrito en el 2007 por Martínez-Calvillo, et al. (19), con algunas modificaciones después de hacer dos lavados de los tripomastigotes con PBS $1 \mathrm{X}$, se volvieron a suspender en solución tampón de lisis $(10 \mathrm{mM}$ Tris- $\mathrm{HCl} \mathrm{pH} 8,0,150 \mathrm{mM} \mathrm{NaCl}$, $0,1 \%$ NP40), teniendo en cuenta que por 1 millón de parásitos se adicionó $1 \mu$ lde tampón de lisis,
Triton X-100 (Sigma; Saint Louis, MO, USA) a una concentración final de 1,4\% y un coctel inhibidor de proteasas (Sigma-Aldrich) a una concentración final de $2 \%$. Los parásitos se incubaron en hielo durante 30 minutos y se centrifugaron a 12.000 rpm durante 15 minutos a $4{ }^{\circ} \mathrm{C}$. La concentración de proteínas del lisado del parásito se determinó por el método de Bradford y el análisis del perfil de proteínas se evaluó en un gel de poliacrilamida (BIORAD) al $10 \%$ con dodecil-sulfato de sodio (SDS-PAGE, Sigma-Aldrich) teñido con nitrato de plata (Sigma-Aldrich) o azul de Coomassie R250 (Gibco BRL; Grand Island, NY, USA) (20).

\section{Curva de producción de citocinas}

Con el fin de determinar el tiempo de cultivo requerido para detectar $\mathrm{LT} \mathrm{CD}^{+}$productores de IFN $\gamma$, IL-2 o TNFa bajo las condiciones experimentales de este estudio, se cultivó un millón de células mononucleares de sangre periférica en $1 \mathrm{ml}$ de medio AIM-V (Gibco) sin estímulo (control negativo), con estímulo policlonal utilizando $1,5 \mu \mathrm{g} /$ $\mathrm{ml}$ de enterotoxina B de Staphylococcus aureus (SEB, Sigma-Aldrich) o con $1 \mu \mathrm{g} / \mathrm{ml}$ de proteínas de lisado de tripomastigotes de T. cruzi como estímulo para evaluar las células específicas en presencia anticuerpos anti-CD28 $(1 \mu \mathrm{g} / \mathrm{ml})$ (BD Biosciences; Catálogo No. 340975) y anti-CD49d $(1 \mu \mathrm{g} / \mathrm{ml})$ (BD Biosciences; Catálogo No. 340976) durante 6, 12, 16 y 20 horas a $37^{\circ} \mathrm{C}$ y CO al $5 \%$. Después de 1,3 , 7 y 11 horas de cultivo, respectivamente, se adicionó el inhibidor de transporte intracelular Brefeldina A (BD GolgiPlug TM; Catálogo No. 555029; San Diego, CA, USA). Una vez terminado el tiempo de cultivo, las células se marcaron con los anticuerpos de superficie y posteriormente se permeabilizaron y fijaron de acuerdo con las instrucciones del estuche comercial (BD Cytofix/Cytoperm ${ }^{\mathrm{TM}}$ Plus; Catálogo No. 555028, San Diego, CA, USA). Finalmente, las células se incubaron con los anticuerpos conjugados anti-citocinas (panel 2): IFN $\gamma$-Alexa Fluor 700 (BD Pharmingen; Clon B27; Catálogo No. 557995), IL-2-PerCP-Cy5.5 (BD Biosciences; Clon MQ1-17H12; Catálogo No. 560708) y TNFaAPC (BD Biosciences; Clon 6401.1111; Catálogo No. 340534) en la oscuridad durante 30 minutos a $4{ }^{\circ} \mathrm{C}$. El estímulo policlonal permite incluir un parámetro de validación para la comparación de las frecuencias obtenidas bajo las mismas condiciones, con las reportadas en otros estudios. El antígeno del parásito permite, además de evaluar la respuesta específica, asegurar la detección de las células de interés para posteriores análisis. 
El panel multicolor para la detección de citocinas en células cultivadas, se evaluó de la misma forma como se describió anteriormente para la marcación en células ex vivo.

\section{Adquisición y análisis de datos por citometría de flujo}

Las lecturas se hicieron en el citómetro de flujo BD FACSAria II (BD Immunocytometry Systems), el cual cuenta con un programa de control de calidad que se hace periódicamente. Con las perlas Cytometer Setup \& Tracking Beads (CS\&T, BD Biosciences; Catálogo No. 642412), se estableció la línea de base del citómetro que permite definir los voltajes de los fotomultiplicadores, así como el alineamiento y el retardo de los láser. Para verificar el correcto funcionamiento del equipo, se estableció día a día la variación de los valores con respecto a la línea de base con las perlas anteriormente mencionadas, en todos los casos con coeficientes de variación menores de $6 \%$ (check perfomance).

Para asegurar la validez y la reproducibilidad de la detección de los paneles utilizados, se aplicaron los mismos parámetros correspondientes a los voltajes de los fotomultiplicadores, umbrales (thresholds) y area scaling a cada experimento, mediante la creación de los parámetros de lectura (application settings), según el boletín técnico de BD Biosciences (21). Posteriormente, para cada lectura se hizo la compensación de las fluorescencias incluidas en el panel multicolor para corregir la posible superposición de los espectros de emisión de los fluorocromos (spillover), utilizando las perlas de captura de anticuerpos CompBeads (BD Biosciences; Catálogo No. 552843). Estas últimas tienen capacidad de unir de manera uniforme a todos los conjugados, de tal forma que permiten una adecuada compensación de las fluorescencias, evitando interferencias en la detección de eventos poco frecuentes. Se utilizaron perlas marcadas con cada fluorocromo y perlas sin marcar, para desarrollar el proceso de la compensación del equipo, calculado automáticamente por el programa de análisis FACSDiva, versión 6.0 (BD Biosciences) $(8,22)$. Una vez calibrado el equipo, se adquirieron al menos 50.000 LT CD8 ${ }^{+}$. Los datos obtenidos se analizaron usando el programa FlowJo, versión 9.3.2 (Tree Star; Ashland, OR, USA).

\section{Resultados}

\section{Selección de moléculas y fluorocromos}

Con el objetivo de evaluar el fenotipo de las diferentes subpoblaciones de $\mathrm{LT} \mathrm{CD8}^{+}$, se seleccionaron las siguientes moléculas: CD3, CD8, CCR7, CD28, CD27, CD45RA, CD95 y CD127. El CCR7 es un receptor de quimiocina expresado en LT vírgenes y células de memoria con un estado de diferenciación temprana, los cuales migran a los órganos linfoides secundarios. CD28 y CD27 son moléculas coestimuladoras y su coexpresión identifica poblaciones de LT CD8 ${ }^{+}$ con diferenciación temprana $\left(\mathrm{CD}^{2} 8^{+}, \mathrm{CD}^{2} 7^{+}\right)$, intermedia (CD28, CD27 ${ }^{+}$) o tardía (CD28, CD27-) (23). CD45RA es una fosfatasa que permite diferenciar LT con fenotipo vírgen y LT activados, y en conjunto con la molécula CCR7, diferencian cuatro subpoblaciones de LT CD8 ${ }^{+}$: LT vírgenes $\left(T_{N}\right)$ $\left(\mathrm{CD} 4 \mathrm{RA}^{+}, \mathrm{CCR}^{+}\right)$, LT de memoria central $\left(\mathrm{T}_{\mathrm{CM}}\right)$ $\left(C D 45 R A, C^{-} C R 7^{+}\right), L T$ de memoria efectora $\left(T_{E M}\right)$ (CD45RA; , CCR7') y LT efectores terminales ( $T_{T E}$ ) (CD45RA $\left.{ }^{+}, \mathrm{CCR}^{-}\right)$(24). CD95 es una molécula expresada en LT activados (25) y, en conjunto con CD45RA, permite la identificación de las células $\mathrm{T}_{\text {scm }}$ (stem cell memory $T$ cells) (26). Finalmente, la disminución de la expresión de CD127 se ha asociado con un mayor grado de diferenciación en $\mathrm{LT} \mathrm{CD8}^{+}(27,28)$.

En el cuadro 1 se muestran los paneles diseñados y las moléculas seleccionadas para la identificación de las subpoblaciones de $\mathrm{LT} \mathrm{CD8}^{+}$en células ex vivo (panel 1) y las subpoblaciones de LT CD8 ${ }^{+}$ productores de IFN $\gamma$, IL-2 y TNFa en células cultivadas (panel 2).

\section{Titulación de anticuerpos conjugados}

La titulación de los anticuerpos se hizo para determinar la concentración óptima del conjugado y minimizarel ruido de fondo. Latitulacióny laselección del punto de saturación se determinaron para cada uno de los conjugados mostrados en el cuadro 1. La figura $1 \mathrm{~A}$ muestra cómo se escogieron los LT $\mathrm{CD}^{+}$para el análisis de moléculas de superficie y la producción de citocinas; se eliminaron las células agrupadas, se seleccionaron las células vivas y la población de linfocitos que expresan CD3 y CD8. La figura 1 muestra un ejemplo de titulación para el anticuerpo conjugado a ficoeritrina-cianina 7 (PECy7) para la evaluación de CCR7 en LT CD8 ${ }^{+}$. Con base en lo recomendado por la casa comercial del conjugado CCR7-PE-Cy7 ( $1 \mu \mathrm{g} / \mathrm{ml})$, se evaluaron las siguientes concentraciones: 0,062, 0,125, 0,25, 0,5 y $1 \mu \mathrm{g} / \mathrm{ml}$, para marcar un millón de células mononucleares de sangre periférica siguiendo las condiciones experimentales y seleccionar el punto de saturación. Cuando las células se marcaron con $0,5 \mu \mathrm{g} / \mathrm{ml}$ del conjugado, la distribución de la 
Cuadro 1. Panel multicolor para la determinación fenotípica y funcional de las subpoblaciones de LT CD8 ${ }^{+}$en células ex vivo y cultivadas

\begin{tabular}{|c|c|c|c|c|c|c|c|c|c|}
\hline \multirow{3}{*}{$\begin{array}{l}\text { Panel } \\
1^{\text {a }}\end{array}$} & \multicolumn{2}{|c|}{ Láser violeta (405 nm) } & \multicolumn{4}{|c|}{ Láser azul (488 nm) } & \multicolumn{3}{|c|}{ Láser rojo $(640 \mathrm{~nm})$} \\
\hline & Amcyam & Pacific Blue & FITC & PE & PerCP-Cy5.5 & PE-Cy7 & Alexa-Fluor700 & APC-H7 & APC \\
\hline & Aqua & CD3 & CD127 & CD45RA & CD28 & CCR7 & CD27 & CD8 & CD95 \\
\hline $2^{\mathrm{b}}$ & Aqua & CD3 & - & CD45RA & IL-2 & CCR7 & $\mathrm{IFN} \gamma$ & CD8 & TNF $\alpha^{a}$ \\
\hline \multicolumn{10}{|c|}{ Panel para la evaluación de las subpoblaciones de LT CD8 ${ }^{+}$en células ex vivo. } \\
\hline
\end{tabular}

${ }^{\text {b }}$ Panel para la determinación de células productoras de IFN $\gamma$, IL-2 y TNF $\alpha$ en subpoblaciones de LT CD8 ${ }^{+}$cultivados con SEB y antígeno crudo de T. cruzi.

A
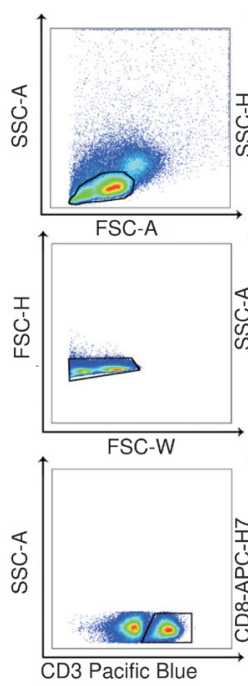
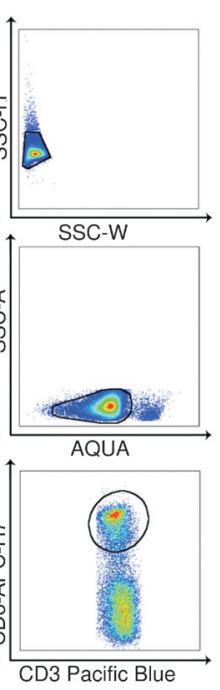

B

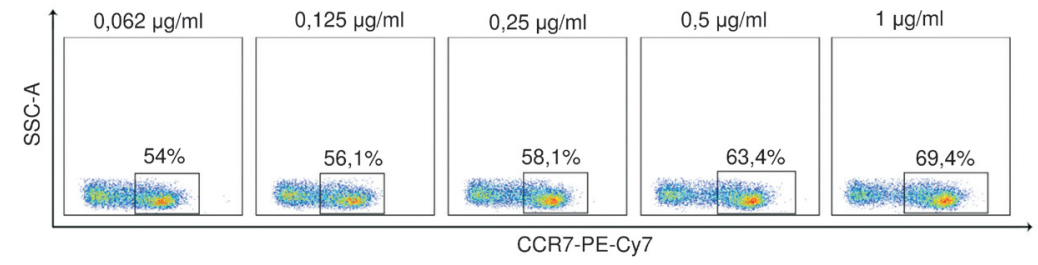

C

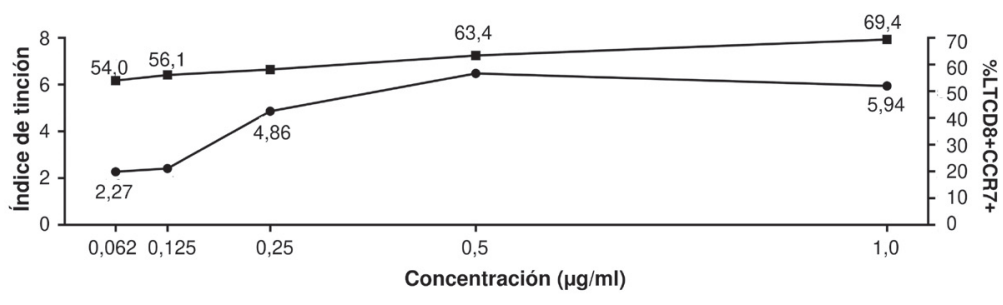

Figura 1. Curva de titulación de conjugados. A). Se seleccionaron las células no agrupadas, vivas y la población de LT que expresan las moléculas CD3 y CD8. B). Dispersogramas representativos de LT CD8 ${ }^{+}$marcados con el conjugado CCR7-PE-Cy7 con las

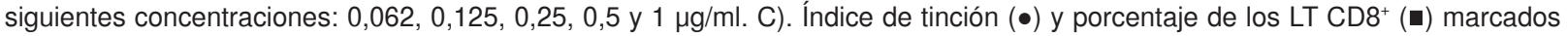
con el conjugado para la identificación de la población $\mathrm{CCR}^{+}$en un individuo sano. El índice de tinción es igual al cociente entre la intensidad media de fluorescencia de la población positiva para el anticuerpo conjugado y la de la población negativa.

población $\mathrm{CCR}^{+}$en $\mathrm{LT} \mathrm{CD8}^{+}$fue adecuada: la población positiva se separa de la negativa y su posición sobre los ejes en los dispersogramas permite identificar los límites de la población de manera clara (figura 1B). Además, cuando las células se marcaron con esta concentración del conjugado, se observó que el índice de tinción correspondía al punto más alto de la curva (figura 1C). Con esta concentración, la frecuencia de LT CD8 ${ }^{+}$positivos para CCR7 fue de $63,4 \%$. En algunos estudios se reportan frecuencias de LT $\mathrm{CD}^{+}{ }^{+} \mathrm{CRR}^{+}$en individuos sanos de $49 \pm 17 \%$ (29) y $55 \%$ (30). Por lo anterior, para este ejemplo el punto de saturación fue $0,5 \mu \mathrm{g} / \mathrm{ml}$, en el cual la concentración fue adecuada para determinar la expresión de LT CD8 ${ }^{+} \mathrm{CCR} 7^{+}$.

\section{Evaluación del panel multicolor}

La evaluación del panel multicolor permite identificar los conjugados de anticuerpos que crean problemas de interferencia alterando su sensibilidad y, además, evalúa el efecto de cada uno de los anticuerpos conjugados sobre la coloración completa $(6,16)$. La evaluación del panel multicolor incluyó la construcción progresiva del panel y la fluorescencia menos uno. Al hacer la construcción progresiva del panel multicolor con los anticuerpos mostrados en el cuadro 1, las frecuencias de las poblaciones no cambiaron, ni alteraron la intensidad media de fluorescencia de cada marcador evaluado a medida que los conjugados se agregaban progresivamente para las células marcadas ex vivo (figura 2). De igual 
manera, la fluorescencia menos uno no mostró disminución de la sensibilidad de los conjugados en el panel para marcar las células ex vivo (figura 3), como tampoco señales de fluorescencia en los canales para los cuales no fue adicionado el conjugado.

\section{Frecuencia de las subpoblaciones de LT CD8} en individuos sanos

Con el fin de utilizar otro parámetro para la evaluación del panel, se determinó la frecuencia de las subpoblaciones de LT CD8 ${ }^{+} T_{N}, T_{S C M}, T_{C M}$, memoria transicional $\left(T_{T M}\right), T_{E M}$ y $T_{T E}$ en individuos sanos utilizando dos (CD45RA y CCR7) y seis moléculas (panel 1, cuadro 1) (figura 4). Según los reportes previos, las subpoblaciones se escogieron con base en la expresión de las moléculas seleccionadas (cuadro 2). Dado que la frecuencia de las subpoblaciones de LT CD8 ${ }^{+}$ evaluadas corresponde a los valores reportados en otros estudios y estas frecuencias dependen del número de moléculas que se utilizaron para el análisis de las subpoblaciones evaluadas, el método propuesto en el presente trabajo asegura la calidad y reproducibilidad de los datos obtenidos (cuadro 3).

\section{Curva de producción de citocinas}

En cuanto a la cinética de producción de IFN $\gamma$, IL-2 y TNFa en células cultivadas con un estímulo policlonal (figura 5A), a las 6 horas laprincipal citocina producida fue TNFa, seguida de IFN $\gamma$ y, por último, IL-2. A las 12 horas de cultivo, la frecuencia de las citocinas evaluadas no varió considerablemente, pero IL-2 mostró su mayor pico de producción a este tiempo. A las 16 horas, mientras la frecuencia

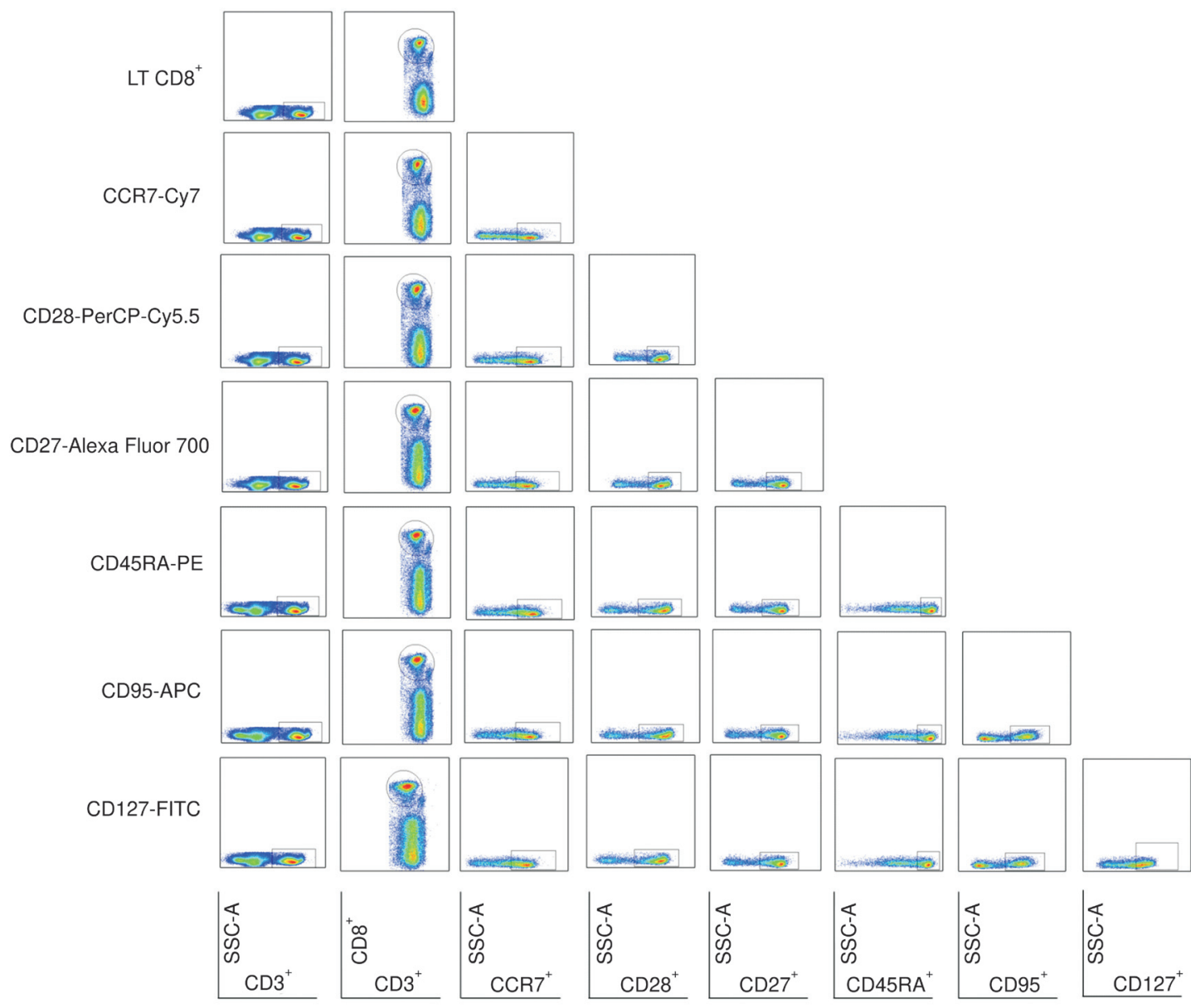

Figura 2. Construcción progresiva de un panel multicolor. El panel multicolor para marcar células ex vivo se construyó con los conjugados para las moléculas CCR7, CD28, CD27, CD45RA, CD95 y CD127, previamente titulados. Las células mononucleares de sangre periférica de un individuo sano se marcaron con los conjugados de manera progresiva, se agregó uno a uno hasta completar el panel. Cada fila representa el número de conjugados que se añadieron. La adición progresiva de los conjugados no mostró cambios en la distribución, frecuencia e intensidad media de fluorescencia de las moléculas analizadas. Los LT CD8 ${ }^{+}$se seleccionaron en cada uno de los casos como se muestra en la figura $1 \mathrm{~A}$. 


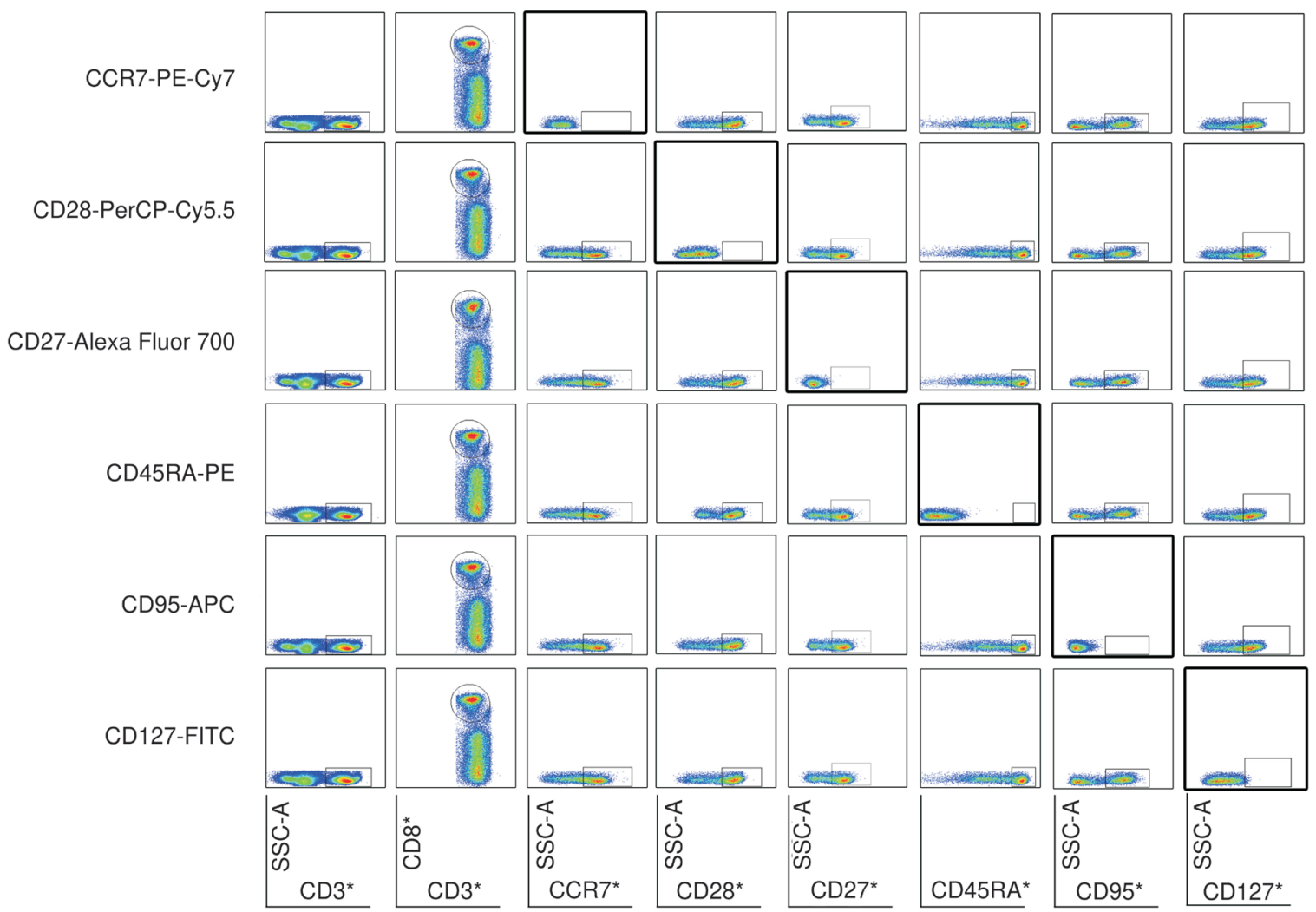

Figura 3. Fluorescencia menos uno. Cada fila representa la población marcada de células ex vivo con el total de conjugados del panel multicolor menos uno. Los dispersogramas resaltados corresponden a la población de células sin el conjugado descrito a la izquierda. En cada uno de los casos, no se observó alteración de la sensibilidad del conjugado que no se adicionó. Los LT CD8 se seleccionaron en cada uno de los casos como se muestra en la figura $1 \mathrm{~A}$.

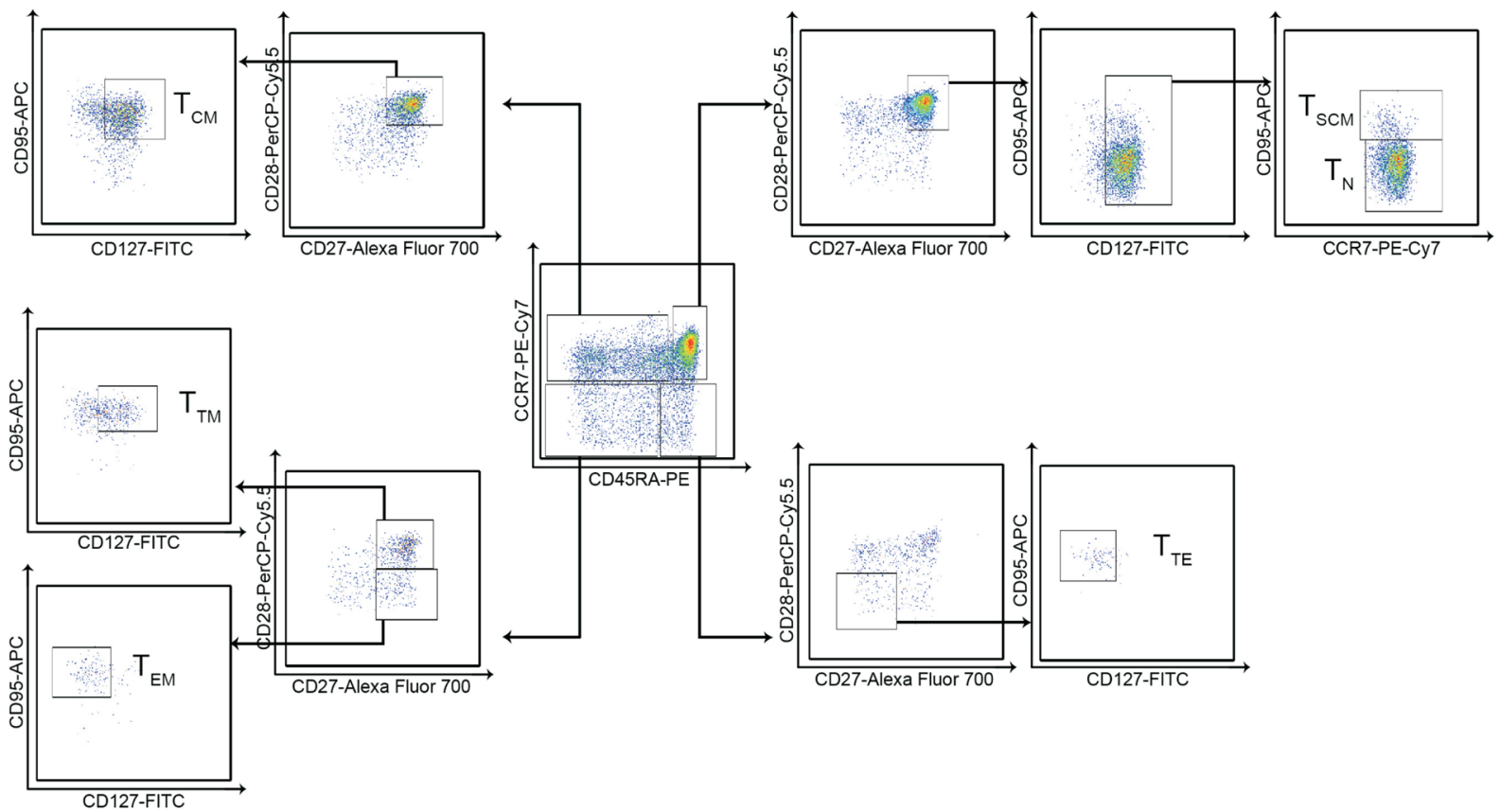

Figura 4. Análisis de las subpoblaciones de $\mathrm{LT} C D 8^{+}$. Los $\mathrm{LT} C D 8^{+}$se seleccionaron como se muestra en la figura $1 \mathrm{~A}$. A partir de estos, se escogieron las subpoblaciones de LT CD8 ${ }^{+}$con dos moléculas (CD45RA y CCR7) y seis moléculas (CCR7, CD28, CD27, CD45RA, CD95 y CD127) en cinco individuos sanos. 
Cuadro 2. Moléculas para la determinación de las subpoblaciones de LT CD8 ${ }^{+}$

\begin{tabular}{lcccccc}
\hline & $\mathbf{T}_{\mathrm{N}}$ & $\mathbf{T}_{\mathrm{SCM}}$ & $\mathbf{T}_{\mathrm{CM}}$ & $\mathbf{T}_{\mathrm{TM}}$ & $\mathbf{T}_{\text {EM }}$ & $\mathbf{T}_{\mathrm{TE}}$ \\
\hline CD45RA & + & + & - & - & - & + \\
CD95 & - & + & + & + & + & + \\
CCR7 & + & + & + & - & - & - \\
CD28 & + & + & + & + & + & - \\
CD27 & + & + & + & + & - & - \\
CD127 & + & + & + & + & $(24)$ & $(24,27,31)$ \\
Referencias & $(24,27,31)$ & $(26)$ & $(24,27,31)$ & & &
\end{tabular}

A

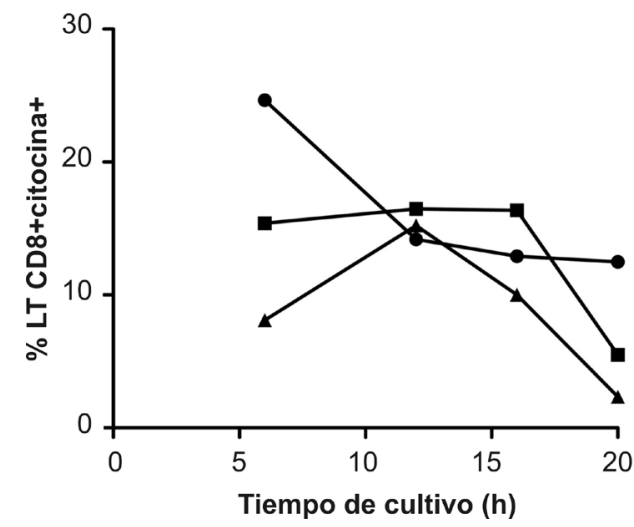

B

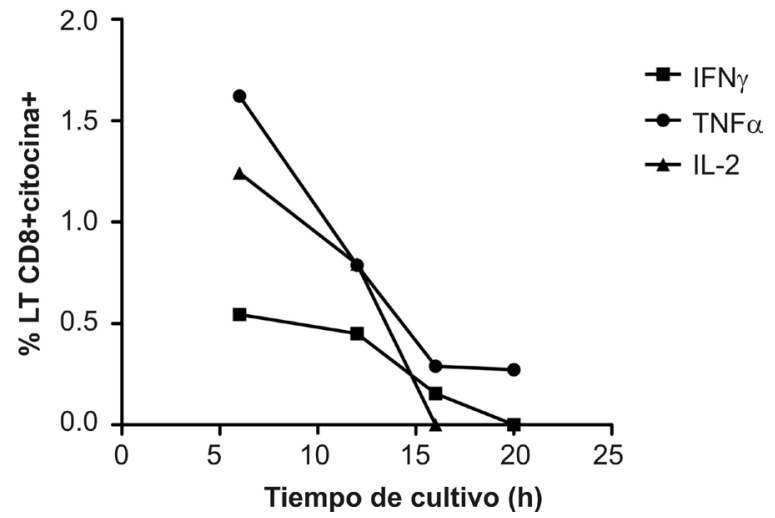

Figura 5. Curva de producción de citocinas intracelulares. Análisis representativo de la frecuencia de LT CD8 ${ }^{+}$que producen IFN $\gamma$, TNFa e IL-2 después del cultivo durante 6, 12, 16 y 20 horas, con un estímulo policlonal (SEB) (A) y específico (antígeno crudo de tripomastigotes de $T$. cruzi) (B), en un paciente con enfermedad de Chagas.

Cuadro 3. Frecuencia de subpoblaciones de $\mathrm{LT} \mathrm{CD8}^{+}$en individuos sanos

\begin{tabular}{lcr}
\hline Fenotipo & Dos moléculas $^{1 *}$ & \multicolumn{1}{c}{ Seis moléculas ${ }^{2 *}$} \\
\hline $\mathrm{T}_{\mathrm{N}}$ & $34,60 \%(27,20-62,90 \%)$ & $18,80 \%(12,10-36,10 \%)$ \\
$\mathrm{T}_{\mathrm{SCM}}$ & - & $2,49 \%(1,96-2,86 \%)$ \\
$\mathrm{T}_{\mathrm{CM}}$ & $25,90 \%(24,20-33,40 \%)$ & $12 \%(9,17-16,80 \%)$ \\
$\mathrm{T}_{\mathrm{TM}}$ & - & $6,22 \%(1,35-9,85 \%)$ \\
$\mathrm{T}_{\mathrm{EM}}$ & $16,10 \%(4,18-27,90 \%)$ & $1,39 \%(0,95-3,38 \%)$ \\
$\mathrm{T}_{\mathrm{TE}}$ & $12 \%(2,33-17,90 \%)$ & $2,16 \%(0,26-3,86 \%)$ \\
\hline $\mathrm{n}=5$ & \\
${ }^{1}=\mathrm{CD} 45 \mathrm{RA}, \mathrm{CCR} 7$. \\
${ }^{2}=\mathrm{CD} 45 \mathrm{RA}, \mathrm{CCR} 7, \mathrm{CD} 28, \mathrm{CD} 27, \mathrm{CD} 95, \mathrm{CD} 127$. \\
${ }^{*}$ Los datos están mostrados en mediana (rango).
\end{tabular}

de células productoras de IFN $\gamma$ se mantuvo, la de las otras citocinas disminuyó. Finalmente, a las 20 horas de cultivo, mientras TNFa se mantuvo, IFN $\gamma$ e IL-2 disminuyeron considerablemente. Para el estímulo específico, a las 6 horas de cultivo se observó la mayor frecuencia de LT $\mathrm{CD}^{+}$productores de las tres citocinas evaluadas, frecuencia que disminuyó gradualmente con el tiempo de cultivo (figura 5B). Este análisis mostró que, bajo las condiciones experimentales utilizadas en la presente nota técnica, en cinco ensayos independientes, se logra una mayor detección de las células productoras de citocinas a las 6 horas de cultivo sin diferencia significativa al comparar con los tiempos evaluados. Además, se valoró el panel para evaluar la producción de citocinas intracelulares (panel 2, cuadro 1) y no se observaron cambios en las frecuencias de las poblaciones, la intensidad media de fluorescencia de las citocinas o la sensibilidad de los fluorocromos con la construcción progresiva del panel multicolor y el control de fluorescencia menos uno (no se presentan los datos).

\section{Discusión}

En la citometría de flujo se han desarrollado varios métodos para marcar antígenos de superficie y antígenos intracelulares, como citocinas, factores de transcripción y proteínas. Muchos fluorocromos con diferentes espectros de excitación y emisión se encuentran disponibles para marcar desde 9 hasta 17 moléculas distintas sobre una célula $(3,5,16)$. En esta nota técnica, se muestra un método para la construcción armónica de un panel multicolor 
con 11 parámetros, que permite evaluar LT CD8 ${ }^{+}$ fenotípica y funcionalmente a partir de células mononucleares de sangre periférica ex vivo y cultivadas. Este método incluye tanto la selección de antígenos y fluorocromos, como la titulación de anticuerpos conjugados y la evaluación de la armonía del panel multicolor.

La titulación de los conjugados tiene el objetivo de minimizar el ruido de fondo, mediante la selección de concentraciones óptimas para la identificación de una molécula (8). El ruido de fondo se ha asociado tanto a falsos positivos como a falsos negativos. La inclusión de un marcador de viabilidad ha mostrado que disminuye el ruido de fondo, dado que las células muertas pueden unirse de manera inespecífica a los anticuerpos conjugados. Por ejemplo, Lamoreaux, et al., mostraron que, con la adición del marcador de viabilidad ViViD en conjunto con la eliminación de poblaciones no deseadas en un canal de descarte (dump channel), se obtiene una frecuencia de LT CD4 ${ }^{+}$que expresan CD107a de $0,061 \%$. Por el contrario, cuando no se incluyen estos parámetros, la frecuencia observada es $5,35 \%$, alejándose significativamente del valor real (8). El canal de descarte corresponde a un grupo de conjugados con un mismo fluorocromo para marcar moléculas en células que no son de interés y descartar poblaciones celulares no deseadas para un análisis más adecuado $(8,17)$. Estas células podrían generar fluorescencia no específica y aumentar el ruido de fondo. La adición del canal de descarte es recomendada cuando se evalúan poblaciones con bajas frecuencias y puede ser agregado en conjunto con el marcador de viabilidad $(17,32)$. Perfetto, et al., recomiendan que, para una mejor detección y discriminación de subpoblaciones de LT, se deben incluir en el canal de descarte conjugados para las moléculas CD19 (linfocitos B), CD56 (células NK) y CD14 (monocitos) (5).

La inclusión de métodos para optimizar un panel multicolor asegura una alta sensibilidad, así como la obtención de datos exactos y reproducibles (5). La sensibilidad de los conjugados con fluorocromos en citometría multiparamétrica está determinada por tres factores: la autofluorescencia de las células, el desempeño del conjugado y la unión de otros conjugados en la misma célula. Para obtener una alta sensibilidad, todos los conjugados deben ser evaluados y analizados para identificar el mejor panel multicolor. Mahnke y Roederer mostraron que el proceso de marcación de moléculas con conjugados puede verse afectado por la presencia de otros conjugados, el orden en el que se agregan, el clon del cual se obtiene el anticuerpo utilizado para identificar una molécula o la superposición de las señales de fluorescencia sobre un mismo detector (spillover). Estos autores mostraron, por ejemplo, que bajo sus condiciones experimentales, la adición del marcador de viablidad ViViD disminuye la sensibilidad del conjugado CD28PE-Cy5 (6). Perfetto, et al., mostraron que el fluorocromo Rojo Texas-Ficoeritrina (TRPE) tiene una gran superposición sobre los canales de PE, PE-Cy5 y PE-Cy5.5 (5). Por otra parte, el control de isotipo es utilizado en la citometría de flujo porque permite determinar el ruido de fondo o la unión inespecífica entre las células y anticuerpos conjugados, haciendo posible diferenciar la población de células negativas de las positivas. En la actualidad, en algunos estudios se recomienda el uso del control de isotipo como control negativo (33). Sin embargo, el uso de este control para determinar antígenos con bajo nivel de expresión o eventos poco frecuentes, no se recomienda porque podría subestimar la población positiva (34).

En 1999, Sallusto, et al., utilizando dos marcadores (CD45RA y CCR7) describieron cuatro subpoblaciones para los LT CD8+: LT vírgenes $\left(T_{N}\right)$, de memoria central $\left(T_{C M}\right)$, memoria efectora $\left(T_{E M}\right)$ y efectoras terminalmente diferenciadas $\left(T_{T E}\right)$ (24). En diferentes estudios se han utilizado estas moléculas para evaluar la frecuencia y función de estas subpoblaciones. Según O'Connor, et al., utilizando CD45RA y CCR7 la frecuencia de las subpoblaciones en $\mathrm{LT} \mathrm{CD}^{+}$es de: 36 a 53,9\% para $\mathrm{T}_{\mathrm{N}}, 14$ a 29,3\% para $\mathrm{T}_{\mathrm{CM}}, 14,3$ a 23,8 \% para $T_{\text {EM }}$ y 10,6 a 20,2 \% para $T_{T E}$ (35). Por otro lado, Austin, et al., mostraron que la frecuencia es para los $T_{N}$ de $41,3 \pm 14,7 \%$, para los $T_{C M}$ de $27,4 \pm 12,6$ $\%$, para los $\mathrm{T}_{\mathrm{EM}}$ de $19,3 \pm 9,8 \%$ y para los $\mathrm{T}_{\mathrm{TE}}$ de $12,1 \pm 10,2 \%$ (36). Por lo tanto, la frecuencia de las subpoblaciones de LT CD8 ${ }^{+}$utilizando CD45RA y CCR7 en estos estudios, es cercana a las frecuencias encontradas en el presente trabajo. No se encontraron estudios donde se evalúen las subpoblaciones de $\mathrm{LT} \mathrm{CD}^{+}$con 6 marcadores. Además, se encontró una amplia variación de la frecuencia de los $T_{E M}$ cuando se evaluaron con dos o seis marcadores. Esto podría explicarse por la presencia de distintos subtipos de $\mathrm{T}_{\mathrm{EM}}$, como los LT de memoria intermedia $0 \mathrm{~T}_{\text {TM }}(27,28,37,38)$. Recientemente, los $\mathrm{T}_{\mathrm{SCM}}$ fueron descritos como una subpoblación de LT distinta de los $T_{N}$ por la expresión de CD95, en un estado de diferenciación temprana con una alta capacidad proliferativa por 
la alta expresión de CD127, CD27 y CD28, y su capacidad de diferenciación en $\mathrm{T}_{C M}$ y $\mathrm{T}_{E M}$. Según Gattinoni, et al., la frecuencia de los $\mathrm{T}_{\mathrm{Scm}}$ en individuos sanos va de 2 a $3 \%$, lo cual corresponde a lo encontrado en el presente trabajo (26).

La detección de la producción de citocinas intracelulares en células cultivadas, depende de las citocinas evaluadas, el estímulo, la población celular de interés y el tiempo de cultivo. El estudio del fenotipo para establecer la subpoblación celular asociado al tipo de citocina producida, permite identificar con mayor precisión el grado de diferenciación celular, tal como se ha descrito anteriormente (39). Además, de acuerdo con los parámetros establecidos en cada modelo experimental, se hace necesario establecer el tiempo adecuado de detección de las citocinas. Por ejemplo, Mascher, et al., determinaron que un cultivo de cinco horas corresponde al mejor momento para evaluar la producción de IFN $\gamma$, IL-2 y TNFa en células mononucleares de sangre periférica estimuladas con fitohemaglutinina (PHA) (15). Rostaing, et al., cuando evaluaron la producción de IFN $\gamma$, IL-2, IL-4, IL-5 e IL-10 en células mononucleares de sangre periférica estimuladas con acetato de forbol miristato (PMA) con ionomicina, encontraron que el tiempo recomendable para evaluar IFN $\gamma$ e IL-2 era de ocho horas de cultivo, en cambio, las demás solo hasta las 36 horas de cultivo (40). Por otro lado, la evaluación del panel multicolor para la detección fenotípica y funcional de LT cultivados, debe hacerse bajo las condiciones experimentales. Con base en esto, aunque existen distintos controles positivos disponibles (SEB, PHA, PMA-ionomicina), es importante evaluar el efecto del estímulo sobre las moléculas y las citocinas que se determinan. Bajo nuestras condiciones experimentales, el tiempo de producción de las citocinas IFN $\gamma$, IL-2 y TNFa se seleccionó con base en el control positivo y en la población de células productoras de las citocinas con el estímulo específico.

En conclusión, el método propuesto para la construcción de un panel multicolor con 11 parámetros para la evaluación fenotípica y funcional de las subpoblaciones de $\mathrm{LT} \mathrm{CD8}^{+}$, no presentó interferencia de los fluorocromos. Este método permite obtener frecuencias de las subpoblaciones de $\mathrm{LT} \mathrm{CD8}{ }^{+}$que corresponden a lo reportado en la literatura científica. Por otro lado, para la evaluación funcional es necesario evaluar bajo las condiciones experimentales del estudio, el tiempo adecuado para revelar la producción de citocinas intracelulares.

\section{Agradecimientos}

A Colciencias y al Programa Jóvenes Investigadores e Innovadores "Virginia Gutiérrez de Pineda".

\section{Conflicto de intereses}

Los autores declaran que no existe conflicto de intereses.

\section{Financiación}

La presente nota técnica fue financiada por la VicerrectoríaAcadémica de la Pontificia Universidad Javeriana en el proyecto No.3333.

\section{Referencias}

1. Bonner WA, Hulett HR, Sweet RG, Herzenberg LA. Fluorescence activated cell sorting. Rev Sci Instrum. 1972;43:404-9. http://dx.doi.org/10.1063/1.1685647

2. Herzenberg LA, Sweet RG, Herzenberg LA. Fluorescenceactivated cell sorting. Sci Am. 1976;234:108-17.

3. De Rosa SC, Herzenberg LA, Herzenberg LA, Roederer M. 11-color, 13-parameter flow cytometry: Identification of human naive $T$ cells by phenotype, function, and T-cell receptor diversity. Nat Med. 2001;7:245-8. http://dx.doi. org/10.1038/84701

4. De Rosa SC, Roederer M. Eleven-color flow cytometry. A powerful tool for elucidation of the complex immune system. Clin Lab Med. 2001;21:697-712.

5. Perfetto SP, Chattopadhyay PK, Roederer M. Seventeencolour flow cytometry: Unravelling the immune system. Nat Rev Immunol. 2004;4:648-55. http://dx.doi.org/10.1038/ nri1416

6. Mahnke YD, Roederer M. Optimizing a multicolor immunophenotyping assay. Clin Lab Med. 2007;27:469-85. http://dx.doi.org/10.1016/j.cll.2007.05.002

7. Roederer M, Brenchley JM, Betts MR, De Rosa SC. Flow cytometric analysis of vaccine responses: How many colors are enough? Clin Immunol. 2004;110:199-205. http://dx.doi. org/10.1016/j.clim.2003.11.015

8. Lamoreaux L, Roederer M, Koup R. Intracellular cytokine optimization and standard operating procedure. Nat Protoc. 2006;1:1507-16. http://dx.doi.org/10.1038/nprot.2006.268

9. Cuellar A, Rojas F, Bolaños N, Diez H, Del Carmen Thomas M, Rosas F, et al. Natural CD4(+) T-cell responses against Trypanosoma cruzi KMP-11 protein in chronic chagasic patients. Immunol Cell Biol. 2009;87:149-53. http://dx.doi.org/10.1038/icb.2008.76

10. Diez H, López MC, Del Carmen Thomas M, Guzmán F, Rosas F, Velazco V, et al. Evaluation of IFN-gamma production by CD8 $\mathrm{T}$ lymphocytes in response to the $\mathrm{K} 1$ peptide from KMP-11 protein in patients infected with Trypanosoma cruzi. Parasite Immunol. 2006;28:101-5. http://dx.doi.org/10.1111/j.1365-3024.2005.00815.x

11. Lasso P, Mesa D, Bolaños N, Cuéllar A, Guzmán F, Cucunubá Z, et al. Chagasic patients are able to respond 
against a viral antigen from influenza virus. BMC Infect Dis. 2012;12:198. http://dx.doi.org/10.1186/1471-2334-12-198

12. Lasso P, Mesa D, Cuéllar A, Guzmán F, Bolaños N, Rosas $\mathrm{F}$, et al. Frequency of specific CD8+ T cells for a promiscuous epitope derived from Trypanosoma cruzi KMP-11 protein in chagasic patients. Parasite Immunol. 2010;32:494-502. http://dx.doi.org/10.1111/j.1365-3024.2010.01206.x

13. Rottenberg ME, Bakhiet M, Olsson T, Kristensson $\mathrm{K}$, Mak T, Wigzell $\mathbf{H}$, et al. Differential susceptibilities of mice genomically deleted of CD4 and CD8 to infections with Trypanosoma cruzi or Trypanosoma brucei. Infect Immun. 1993;61:5129-33.

14. Wizel B, Palmieri M, Mendoza C, Arana B, Sidney J, Sette A, et al. Human infection with Trypanosoma cruzi induces parasite antigen-specific cytotoxic T lymphocyte responses. J Clin Invest. 1998;102:1062-71. http://dx.doi.org/10.1172/ $\mathrm{JCl} 3835$

15. Mascher B, Schlenke P, Seyfarth M. Expression and kinetics of cytokines determined by intracellular staining using flow cytometry. J Immunol Methods. 1999;223:11521. http://dx.doi.org/10.1016/S0022-1759(98)00200-2

16. McLaughlin BE, Baumgarth N, Bigos M, Roederer M, De Rosa SC, Altman JD, et al. Nine-color flow cytometry for accurate measurement of $\mathrm{T}$ cell subsets and cytokine responses. Part I: Panel design by an empiric approach. Cytometry A. 2008;73:400-10. http://dx.doi.org/10.1002/ cyto.a.20555

17. Baumgarth N, Roederer M. A practical approach to multicolor flow cytometry for immunophenotyping. J Immunol Methods. 2000;243:77-97. http://dx.doi.org/10.1016/S00221759(00)00229-5

18. Yoshida N, Mortara RA, Araguth MF, González JC, Russo M. Metacyclic neutralizing effect of monoclonal antibody 10D8 directed to the 35- and 50-kilodalton surface glycoconjugates of Trypanosoma cruzi. Infect Immun. 1989;57:1663-7.

19. Martínez-Calvillo S, Saxena A, Green A, Leland A, Myler PJ. Characterization of the RNA polymerase II and III complexes in Leishmania major. Int J Parasitol. 2007;37:491502. http://dx.doi.org/10.1016/j.ijpara.2006.11.019

20. Sambrook JF, Russell DW. Molecular cloning, a laboratory manual. 3rd edition. New York: Cold Spring Harbor Laboratory; 2001.

21. Meinelt E, Reunanen M, Edinger M, Jaimes M, Stall A, Sasaki D, et al. Standardizing application setup across multiple flow cytometers using BD FACSDivaTM Version 6 Software: Technical Bulletin. Piscataway: BD Biosciences; 2012. p.16

22. Roederer M. Spectral compensation for flow cytometry: Visualization artifacts, limitations, and caveats. Cytometry. 2001;45:194-205.http://dx.doi.org/10.1002/10970320(20011101)45:3<194::AID-CYTO1163>3.0.CO;2-C

23. Appay V, Dunbar PR, Callan M, Klenerman $P$, Gillespie GM, Papagno L, et al. Memory CD8+ T cells vary in differentiation phenotype in different persistent virus infections. Nat Med. 2002;8:379-85. http://dx.doi. org/10.1038/nm0402-379

24. Sallusto F, Lenig D, Forster R, Lipp M, Lanzavecchia A. Two subsets of memory T lymphocytes with distinct homing potentials and effector functions. Nature. 1999;401:708-12. http://dx.doi.org/10.1038/44385

25. Cossarizza A, Stent G, Mussini C, Paganelli R, Borghi V, Nuzzo C, et al. Deregulation of the CD95/CD95L system in lymphocytes from patients with primary acute HIV infection. AIDS. 2000;14:345-55.

26. Gattinoni L, Lugli E, Ji Y, Pos Z, Paulos CM, Quigley MF, et al. A human memory $T$ cell subset with stem celllike properties. Nat Med. 2011;17:1290-7. http://dx.doi. org $/ 10.1038 / \mathrm{nm} .2446$

27. Appay V, van Lier RA, Sallusto F, Roederer M et al Phenotype and function of human T lymphocyte subsets: Consensus and issues. Cytometry A. 2008;73:975-83. http://dx.doi.org/10.1002/cyto.a.20643

28. Burgers WA, Riou C, Mlotshwa M, Maenetje P, de Assis RD, Brenchley J, et al. Association of HIV-specific and total CD8+ T memory phenotypes in subtype C HIV-1 infection with viral set point. J Immunol. 2009;182:4751-61. http:// dx.doi.org/10.4049/jimmunol.0803801

29. Kim JW, Ferris RL, Whiteside TL. Chemokine $C$ receptor 7 expression and protection of circulating CD8+ Tlymphocytes from apoptosis. Clin Cancer Res. 2005;11:7901-10. http:// dx.doi.org/10.1158/1078-0432.CCR-05-1346

30. Payne D, Drinkwater S, Baretto R, Duddridge M, Browning MJ. Expression of chemokine receptors CXCR4, CXCR5 and CCR7 on $B$ and $T$ lymphocytes from patients with primary antibody deficiency. Clin Exp Immunol. 2009;156:254-62. http://dx.doi.org/10.1111/j.1365-2249.2009.03889.x

31. Sallusto F, Geginat J, Lanzavecchia A. Central memory and effector memory $T$ cell subsets: Function, generation, and maintenance. Annu Rev Immunol. 2004;22:745-63. http://dx.doi.org/10.1146/annurev.immunol.22.012703. 104702

32. Perfetto SP, Chattopadhyay PK, Lamoreaux L, Nguyen R, Ambrozak D, Koup RA, et al. Amine reactive dyes: An effective tool to discriminate live and dead cells in polychromatic flow cytometry. J Immunol Methods. 2006;313:199-208. http://dx.doi.org/10.1016/j. jim.2006.04.007

33. O'Gorman MR, Thomas J. Isotype controls -time to let go? Cytometry. 1999;38:78-80. http://dx.doi. org/10.1002/(SICI)1097-0320(19990415)38:2<78::AIDCYTO6>3.0.CO;2-E

34. Keeney M, Gratama JW, Chin-Yee IH, Sutherland DR. Isotype controls in the analysis of lymphocytes and CD34+ stem and progenitor cells by flow cytometry -time to let go! Cytometry. 1998;34:280-3. http://dx.doi. org/10.1002/(SICI)1097-0320(19981215)34:6<280::AIDCYTO6>3.0.CO;2-H

35. O'Connor AM, Crawley AM, Angel JB. Interleukin-7 enhances memory CD8(+) T-cell recall responses in health but its activity is impaired in human immunodeficiency virus infection. Immunology. 2010;131:525-36. http://dx.doi. org/10.1111/j.1365-2567.2010.03325.x

36. Austin ED, Rock MT, Mosse CA, Vnencak-Jones CL, Yoder SM, Robbins IM, et al. T lymphocyte subset abnormalities in the blood and lung in pulmonary arterial hypertension. Respir Med. 2010;104:454-62. http://dx.doi. org/10.1016/j.rmed.2009.10.004 
37. Romero P, Zippelius A, Kurth I, Pittet MJ, Touvrey C, lancu EM, et al. Four functionally distinct populations of human effector-memory CD8+ T lymphocytes. J Immunol. 2007;178:4112-9.

38. Takata $\mathbf{H}$, Takiguchi $\mathbf{M}$. Three memory subsets of human CD8+ T cells differently expressing three cytolytic effector molecules. J Immunol. 2006;177:4330-40.

39. Seder RA, Darrah PA, Roederer M. T-cell quality in memory and protection: Implications for vaccine design. Nat Rev Immunol. 2008;8:247-58. http://dx.doi.org/10.1038/nri2274
40. Rostaing L, Tkaczuk J, Durand M, Peres C, Durand D, de Preval C, et al. Kinetics of intracytoplasmic Th1 and Th2 cytokine production assessed by flow cytometry following in vitro activation of peripheral blood mononuclear cells. Cytometry. 1999;35:318-28. http://dx.doi. org/10.1002/(SICl)1097-0320(19990401)35:4<318::AIDCYTO4>3.0.CO;2-4 